\title{
Lutz Maeke, DDR und PLO - Die Palästinapolitik des SED-Staates
}

Dominique Trimbur

\section{OpenEdition}

Journals

Édition électronique

URL : http://journals.openedition.org/ifha/9014

DOI : 10.4000/ifha.9014

ISSN : 2198-8943

Éditeur

IFRA - Institut franco-allemand (sciences historiques et sociales)

Référence électronique

Dominique Trimbur, "Lutz Maeke, DDR und PLO - Die Palästinapolitik des SED-Staates », Revue de l'IFHA [En ligne], Date de recension, mis en ligne le 28 mars 2018, consulté le 24 septembre 2020. URL : http://journals.openedition.org/ifha/9014 ; DOI : https://doi.org/10.4000/ifha.9014

Ce document a été généré automatiquement le 24 septembre 2020

(C)IFHA 


\title{
Lutz Maeke, DDR und PLO - Die Palästinapolitik des SED-Staates
}

\author{
Dominique Trimbur
}

\section{RÉFÉRENCE}

Lutz Maeke, DDR und PLO - Die Palästinapolitik des SED-Staates, Berlin/Boston: De Gruyter/ Oldenbourg (Wissenschaftliche Reihe des Fritz-Bauer-Instituts), 2017, 548 p., 79,95€ 
L'ouvrage de Lutz Maeke revient sur un aspect à la marge, mais important et intéressant, des relations germanoisraéliennes, désormais largement balisées, et du rapport de l'Allemagne au Moyen-Orient, lui aussi largement exploré. La majeure partie des études disponibles insistent sur la constellation qui met en rapport la RFA (avant et après 1990) avec Israël et le Moyen-Orient. L. Maeke, lui, se penche sur la RDA et sa politique palestinienne. Des études anciennes, notamment celles d'Angelika Timm ou de Michael Wolffsohn, ont exploré le champ, mettant en avant les relations entre la RDA et Israël, évoquant en creux l'attitude de Berlin-Est à l'égard de la question palestinienne. D'autres plus récentes (Jeffrey Herf) se sont intéressées au sujet, prenant prétexte de l'analyse historique pour constituer un dossier à charge, pointant du doigt les "guerres non déclarées » de la RDA à l'encontre d'Israël et encourant le risque d'une simplification extrême. Sur cette base, L. Maeke reprend le dossier en se voulant quasi exhaustif. La finitude de son sujet le lui permet, la RDA ayant cessé d'exister, ce qui justifie un bornage chronologique bien déterminé : 1949-1990, et plus précisément 1964-1990, à partir de la création de l'OLP.

À l'appui de nombreuses archives, notamment celles du ministère de Sécurité d'Etat (la "Stasi»), L. Maeke retrace une histoire plus complexe que ce qui avait été décrit jusqu'ici. Il demeure avéré que, globalement, la RDA a eu une attitude pour le moins défiante envers Israël et a témoigné d'une vraie empathie envers la cause palestinienne. Mais ce partage entre inimitié et amitié n'est pas complètement cloisonné ; surtout, la nature du soutien à la cause palestinienne doit être nuancée, même si la RDA a résolument appuyé les factions palestiniennes les plus farouches, validant ainsi leur recours systématique au terrorisme.

L'expression «à nouveaux frais » s'applique particulièrement bien ici. Contrairement à ce que l'on croyait savoir, il faut par exemple attendre la fin des années 1960 pour que se mette en place une politique palestinienne de la RDA à proprement parler. Jusque-là, cette dernière s'attache au respect des résolutions de l'ONU : certes Berlin-Est s'aligne sur une URSS antisioniste (voire antisémite), mais, dans les années 1950, la RDA considère que les aspirations respectives doivent être respectées et qu'Israël a le droit d'exister. Un respect qui s'explique aussi par une véritable méfiance envers la cause palestinienne qui s'organise progressivement : visant à une libération nationale, cette cause a priori respectable semble par trop nationaliste et teintée de religion; de plus, elle est appuyée par des États arabes réputés « réactionnaires ». En tant que telle, l'OLP est créée en 1964, mais elle n'en devient pas pour autant automatiquement l'interlocutrice privilégiée. Si des échanges se mettent en place sous la forme 
d'assistance (accueil d'étudiants, d'orphelins...), ces derniers passent par des officines semi-étatiques, et non par le ministère des Affaires étrangères; et si l'OLP est impliquée, on ne lui reconnaît pas une nature étatique, non plus que l'on ne valorise le Fatah et son leader, Yasser Arafat (qui prend la tête de l'OLP en 1968). C'est seulement à partir de la guerre des Six jours qu'une prise en considération plus grande de la cause palestinienne s'effectue: Israël ayant confirmé son appartenance au camp des pays " impérialistes" par son "agression ", il est désormais davantage possible d'afficher son appui aux États arabes et à la défense de cette cause, devenue entre-temps plus révolutionnaire et dont l'idéologie puise désormais aux sources du marxisme-léninisme cher aux responsables est-allemands.

Les années 1970 constituent l'âge d'or de la cause palestinienne; pour des raisons néfastes, le terrorisme palestinien multipliant ses coups d'éclat, et pour des raisons plus positives, l'OLP obtenant une reconnaissance internationale. La RDA accompagne ce mouvement, procurant une assistance militaire à l'OLP, acceptant d'inviter Arafat à Berlin-Est (en 1971, puis aux Jeux mondiaux de la jeunesse, en 1973) et accordant à son organisation l'installation d'une représentation (sous la forme d'un bureau en 1973, puis d'une représentation en 1978). L. Maeke rappelle toutefois que la RDA avance à un rythme moins soutenu que la RFA dans les faveurs accordées à la cause palestinienne : le bureau palestinien ouvert à Bonn (1970) précède celui de Berlin-Est, et, parallèlement à sa politique généralement pro-israélienne, l'Allemagne de l'Ouest agit pour faire avancer la cause palestinienne dans le cadre européen ou dans celui de l'Internationale socialiste (W. Brandt).

Ces faveurs occidentales constituent autant d'éléments qui vont finir par froisser Berlin-Est. La promotion de la cause palestinienne peut, à ses yeux, se passer d'un appui au leader charismatique : le meilleur allié du camp soviétique au Moyen-Orient est alors la Syrie d'Hafez el Assad, lui-même farouchement opposé à Arafat, et dont la violence sanglante envers l'OLP (au Liban en 1976, puis en 1982-83) ne suscite aucune objection à Berlin-Est. C'est à ce moment que se renforce le soutien apporté par la RDA aux groupuscules «progressistes " rivaux du Fatah, idéologiquement plus proches d'elle (FDLP, FPLP) et plus manipulables par la Stasi, partisans fanatiques de la destruction d'Israël et de l'action terroriste. Confirmant sa méfiance envers un Arafat trop porté au rapprochement avec les Occidentaux, qui sont davantage en mesure de conforter la reconnaissance internationale de la cause palestinienne, la RDA abrite et fournit en armes le groupe d'Abou Nidal, et n'intervient pas pour freiner des attaques antiaméricaines à Berlin-Ouest organisées à partir de bases arrières établies dans l'Est de la ville (attentat contre la discothèque « La Belle » en avril 1986, en lien avec les services libyens).

Comme le montre L. Maeke, c'est seulement contraint et forcé que le régime de BerlinEst va réorienter sa politique. Dans le contexte de la politique de détente de Gorbatchev se dessine en effet un assouplissement de l'attitude communiste envers Israël. Honecker, soucieux d'un rapprochement très intéressé avec les États-Unis, entreprend une politique pro-juive (prise en compte de la responsabilité issue du passé nazi et perspective de dédommagements aux Juifs, jamais sérieusement envisagés jusque-là) dans l'objectif d'améliorer l'image de la RDA. Somme toute vaine, cette politique implique l'abandon des relations nouées avec les groupuscules palestiniens radicaux, et donc la reconnaissance d'un Arafat qui a su affirmer son leadership sur le mouvement palestinien et s'imposer comme l'unique interlocuteur. Cette évolution ressemble bien à un Canossa pour Berlin-Est, comme le marque la remise à Arafat de la plus haute 
décoration est-allemande en... octobre 1989. Mais Arafat peut agir sereinement: ses principales avancées se font finalement sans consulter ceux qu'on pourrait continuer de percevoir comme ses alliés naturels, le bloc communiste et en particulier la RDA. Ayant joué sur les deux tableaux, il semble, en tout cas à ce stade, avoir réussi son pari et rendu fréquentable la cause palestinienne, la Cisjordanie étant désormais envisagée comme l'espace où pourrait se réaliser l'État de Palestine, qu'il proclame symboliquement en 1988.

Quelques pages en fin de volume permettent d'évoquer l'évolution ultime d'une RDA encore communiste puis véritablement démocratique: engagement de pourparlers avec Israël en vue de réparations accordées aux Juifs et de relations diplomatiques avec Israël. Mais la RDA cesse d'exister avant que des engagements soient scellés. L'Allemagne réunifiée permet toutefois de concrétiser les perspectives dessinées alors, tandis que pour Arafat, elle peut contribuer à la réussite de la cause qu'il incarne.

L. Maeke est convaincant, même si on peut lui reprocher d'insister sur la justesse de ses vues, il est vrai appuyées par les ressources archivistiques, en contrepoint de ses prédécesseurs ayant travaillé sur le sujet; et même si son approche lui fait comparer des situations non équivalentes (une relation à un État comme la Syrie peut-elle être comparée à l'attitude envers une organisation incarnant ce qui n'est qu'un mouvement national ?). Au-delà des clichés ou des visions trop simplificatrices qu'il chamboule (pas de rôle de la RDA dans la défense de la cause palestinienne, l'appui ouest-allemand à l'OLP est bien plus important dans la même période), il parvient, en dépit d'une écriture pas toujours aisée, à démontrer que la constellation qu'il réexplore est plus complexe que ce que l'on pensait jusque-là. À ce titre, le livre de L. Maeke pourra faire figure de référence.

\section{INDEX}

Index chronologique : Époque contemporaine

Thèmes : Histoire des États et des pouvoirs

\section{AUTEUR}

\section{DOMINIQUE TRIMBUR}

Chercheur associé au Centre de Recherche Français de Jérusalem 